\title{
THE CMB - CONTEMPORARY MEASUREMENTS AND COSMOLOGY
}

\author{
A. D. MILLER* \\ Department of Astronomy and Astrophysics, The University of Chicago, $5640 \mathrm{~S}$. \\ Ellis Ave., Chicago, IL 60637, USA \\ E-mail: amber@oddjob.uchicago.edu
}

\begin{abstract}
Since the discovery of the Cosmic Microwave Background (CMB) in 1965, characterization of the CMB anisotropy angular power spectrum has become somewhat of a holy grail for experimental cosmology. Because CMB anisotropy measurements are difficult, the full potential of the CMB is only now being realized. Improvements in experimental techniques and detector technology have yielded an explosion of progress in the past couple of years resulting in the ability to use measurements of the CMB to place meaningful constraints on cosmological parameters. In this review, I discuss the theory behind the CMB but focus primarily on the experiments, reviewing briefly the history of $\mathrm{CMB}$ anisotropy measurements and focusing on the recent experiments that have revolutionized this field. Results from these modern experiments are reviewed and the cosmological implications discussed. I conclude with brief comments about the future of CMB physics.
\end{abstract}

\section{Introduction}

The Cosmic Microwave Background (CMB) consists of photons emitted in the early hot, dense phase of the Universe before the formation of structure by gravitational collapse. CMB photons are the oldest remaining photons in the universe; in effect a snapshot of the initial conditions for structure formation. The CMB is therefore a valuable tool for connecting the structure we see in the universe today with theories predicting its origin.

At times earlier than $z \approx 1000$, the universe consisted of a hot dense plasma in which electrons were electromagnetically coupled to baryons, photons were coupled to electrons via Compton scattering, and the universe was opaque to radiation. When the universe cooled enough to form the first neutral hydrogen atoms, the number density of free-electron scatterers dropped precipitously and the photons effectively decoupled from the baryons. Small inhomogeneities in the initial density field, which existed before decoupling, seeded the gravitational collapse of baryonic mater, resulting in the formation of all observable structures in the current universe. The photons were left to free stream and cool, interacting very little for the remaining history of the universe. These CMB photons therefore provide the cleanest known

${ }^{*}$ Hubble Fellow 
probe of the early universe; anisotropies in the observed CMB temperature field directly correspond to the tiny fluctuations in the initial density field.

\section{Anisotropy in the CMB - Theoretical Overview}

The CMB is remarkably uniform, but small inhomogeneities in the density field of the early universe are revealed by temperature fluctuations of order one part in $10^{5}$ in the currently observable CMB. Information from measurements of these fluctuations is quantified by constructing the angular power spectrum of the fluctuations discussed below. Since different models of structure formation predict different power spectrum characteristics, observations can be used to test cosmological predictions.

\subsection{The Angular Power Spectrum}

CMB temperature fluctuations on the sky can be described by an expansion in spherical harmonics,

$$
T(\theta, \phi)=\sum_{l, m} a_{l m} Y_{l m}(\theta, \phi) .
$$

For Gaussian fluctuations, the multipole moments of the temperature field are fully described by their angular power spectrum,

$$
\left\langle a_{l m}^{*} a_{l \prime m \prime}\right\rangle=\delta_{l l \prime} \delta_{m m \prime} C_{l}
$$

The angular power spectrum can be related to the autocorrelation function, $C(\theta)$, as

$$
C(\theta)=\frac{1}{4 \pi} \sum_{l}(2 l+1) C_{l} P_{l}(\cos \theta)
$$

where the $P_{l}$ 's are the Legendre Polynomials. Since the Legendre Polynomials are orthogonal, the individual values of $C_{l}$ can be recovered by multiplying $C(\theta)$ by a Legendre Polynomial and integrating over $\cos \theta$.

It is customary to plot the angular power spectrum, $C_{l}$, in terms of $\left(\delta T_{l}\right)^{2}$, the power per logarithmic interval in $l$,

$$
\left(\delta T_{l}\right)^{2}=\frac{1}{2 \pi} l(l+1) C_{l}
$$

or as the square root of this quantity,

$$
\delta T_{l}=\sqrt{\frac{l(l+1) C_{l}}{2 \pi}} .
$$




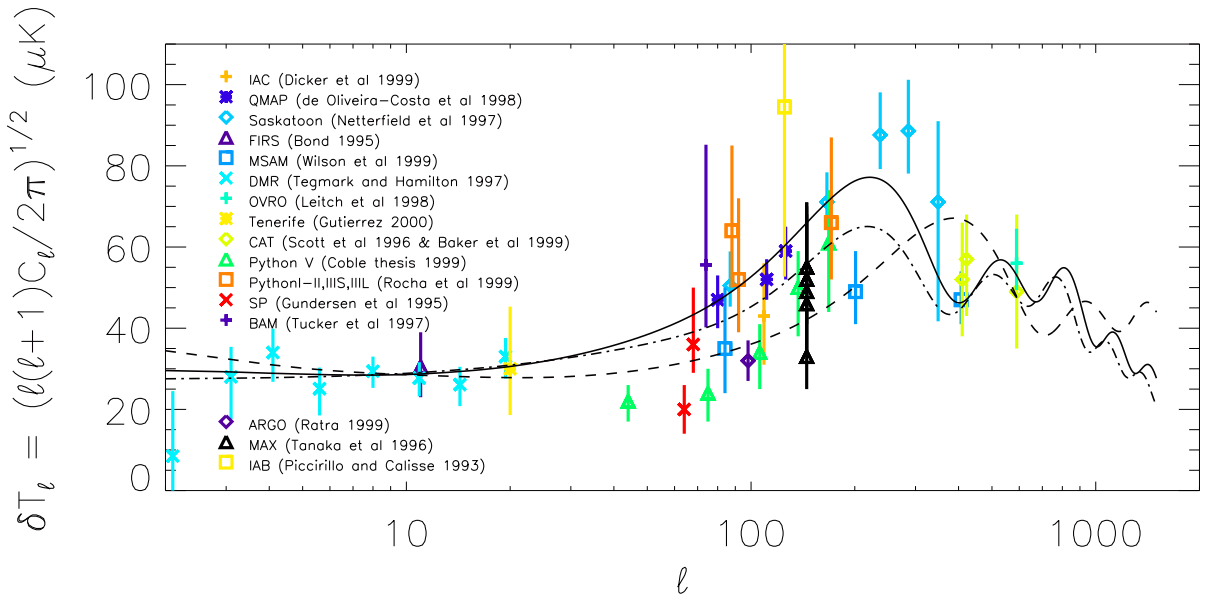

Figure 1. Models for the CMB angular spectrum computed with CMBfast 73 plotted with CMB data published as of 1999. The solid line is a $\Lambda$ CDM model with $\Omega_{m}=0.33, \Omega_{b}=$ $0.041, \Omega_{\Lambda}=0.67$, and $h=0.65$. The dotted line is a standard CDM model with $\Omega_{m}=1$, $\Omega_{b}=0.05$, and $h=0.65$. The dashed line is an open model with $\Omega_{m}=0.3, \Omega_{b}=0.05$, and $h=0.65$. Plotted CMB bandpowers do not include calibration errors, which are typically at least $10-20 \%$.

\subsection{Primary Anisotropies}

Three representative models for the CMB angular spectrum are shown in Figure1. A typical model is characterized by a flat plateau at large angular scales rising with $l$ to a first prominent peak, and followed by peaks of decreasing amplitude at smaller angular scales. The series of peaks and valleys is referred to as "Doppler" or "acoustic" peaks. The primary anisotropies discussed in this section are those occurring on the surface of last scattering. We distinguish them from secondary anisotropies discussed in the next section, which are due to photons scattering along the line of sight between the surface of last scattering and the observer. The three fundamental effects that produce primary anisotropies can be summarized by the equation 5268 ,

$$
\frac{\delta T}{T}(\mathbf{r})=\phi(\mathbf{r})+\frac{1}{3} \delta(\mathbf{r})-\delta \mathbf{v} \cdot \hat{\mathbf{r}} .
$$

The first term represents a redshift due to the gravitational potential, $\phi$. Photons in overdense regions must climb out of potential wells in order 
to reach an observer. Dense regions, therefore appear cooler by an amount, $\delta T / T=\phi(\mathbf{r})$.

The second term is the adiabatic contribution. The physical mechanism behind this term is presented by $\mathrm{Hu}$, Sugiyama, and Silk 31 and is explained in detail there. The basic idea is the following. Before decoupling, the photons and baryons are tightly coupled (due to Compton scattering between electrons and photons and Coulomb interactions between electrons and baryons) and can be treated as a single fluid. In the context of a cold dark matter (CDM) model, Gaussian random fluctuations in the dominant CDM component create potential wells into which the photon-baryon fluid falls. The potential wells compress the fluid, the photon temperature increases, and the radiation pressure rises enough to overcome the compression and to cause a rarefaction. These oscillations proceed until recombination when the photon-baryon coupling breaks down. The denser regions on the last scattering surface will therefore be hotter while the less dense regions are cooler. Following inflation but before recombination, the horizon size increases and oscillation modes of increasing size enter the horizon. These modes are frozen in at last scattering, leaving peaks in the angular spectrum corresponding to modes caught at extrema at that epoch. The smallest wavelength modes have had the most time to evolve before being frozen. Odd peaks represent the compression maxima while even ones result from rarefaction maxima. The first "Doppler Peak" represents the mode that has just reached its first compression maximum at the time of last scattering. The density perturbations can be parameterized in terms of the density contrast,

$$
\delta=\frac{\delta \rho}{\rho},
$$

where $\rho$ is the average density and $\delta \rho$ is the local density perturbation. Assuming that the universe was matter-dominated at the epoch of decoupling, the density $\rho \propto T^{3}$ so

$$
\frac{\delta T}{T \text { adiabatic }}=\frac{1}{3} \delta .
$$

The longest wavelength modes ( $>$ a few degrees) will not have had time to evolve significantly by the time of decoupling and will yield information about the primordial potential fluctuations. Assuming adiabatic initial conditions, $\delta \approx-2 \phi 30$, or

$$
\frac{\delta T}{T}_{\text {adiabatic }}=-\frac{2}{3} \phi
$$


In this case, the first and second terms partially cancel yielding

$$
\frac{\delta T}{T}=\frac{\phi}{3} .
$$

This is called the Sachs-Wolfe effect and is responsible for the plateau in the angular spectrum seen in Figure 1 at large angular scales (low $l$ ).

The third term represents the Doppler contribution, which is due to the combination of the Earth's motion with respect to the CMB and the motion of the matter on the surface of last scattering. In a universe in which the baryon contribution is large enough to be consistent with nucleosynthesis, the velocity contribution is subdominant 30 .

\subsection{Secondary Anisotropies}

In addition to the primary anisotropies that imprint temperature fluctuations on the CMB before decoupling, several processes also exist after last scattering that imprint detectable secondary anisotropies. These can be broadly divided into two categories; those due to gravitational effects (early and late Integrated Sachs-Wolfe (ISW) effects and the Rees-Sciama effect), and those due to reionization at later times in the history of the universe. The megh anisms governing secondary CMB anisotropies are discussed elsewhere 30.68 but some observational consequences of these effects are presented in the next section.

\subsection{Observational Signatures}

Measurements of the CMB angular spectrum are a powerful probe of the underlying cosmology because the detailed shape of the power spectrum depends on the specifics of the cosmological model. In particular, parameters such as the energy density of the universe relative to the critical value, $\Omega$, the fraction of that density in baryons, $\Omega_{b}$, matter, $\Omega_{m}$, curvature, $\Omega_{k}$ and due to a cosmological constant, $\Omega_{\Lambda}$, as well as the Hubble constant, $H_{0}$, the tilt, $n$, and the reionization fraction, $\tau$ each leave an observable signature on the CMB angular power spectrum. Discussed below are some of the cosmological parameters which, according to the most popular class of adiabatic models, can be estimated by careful measurement of the angular power spectrum.

- $\Omega_{k}$ : The location of the acoustic peaks in $l$-space depends most sensitively on the curvature of the universe through a simple projection effect 33 . The scale of the sound horizon in the photon-baryon fluid before decoupling sets the maximum size of a causally connected region, corresponding to the angular size of the first acoustic peak. The radiation density is fixed by the

miller: submitted to World Scientific on November 27, 2018 
FIRAS measurements of $\mathrm{T}_{\mathrm{CMB}}$ today 21 so the physical size of the sound horizon depends primarily on $\Omega_{m} h^{2}$ and can be used as a standard ruler. The measured angular scale at which this peak is detected therefore sheds light on the background geometry. The measured angular scale for an object of fixed size will be larger for a universe with positive curvature than for a flat universe and will be smaller for a universe with negative curvature. When detailed calculations are performed, the first acoustic peak is shown to be located at $l \approx 220$ for a large class of flat models and $l \approx 350-400$ for a large class of open models.

- $\Omega_{m}$ : The primary signature on the CMB of the matter density is a shift in the position of the peak due to the curvature effect discussed above. A lower value of $\Omega_{m}$ in the absence of a compensating higher value of $\Omega_{\Lambda}$ will shift the peak to higher $l$ values. In the context of a flat universe, a lower value of $\Omega_{m}$ implies a larger $\Omega_{\Lambda}$ or $\Omega_{D E}$.

- $\Omega_{\Lambda}$ or $\Omega_{D E}$ : If the universe is dominated by a negative pressure component such as a cosmological constant or some other form of dark energy, the lowest multipoles will be enhanced by the late ISW effect. The presence of a negative pressure component provides the necessary energy density to accommodate a flat universe in which $\Omega_{m} \approx 0.3$, consistent with other measurements 22. In a flat universe, the early ISW effect leads to an increase in amplitude of the first peak with increasing cosmological constant. This dependence is due to the fact that the baryon-to-photon ratio before decoupling is lower in models that are dominated at the present epoch by some form of dark energy than it is in flat models in which the energy density is due entirely to matter. This is the same effect as described above for a lower value of $\Omega_{m}$ but peak amplitude is boosted without the accompanying shift to smaller angular scales characteristic of open models.

- $\Omega_{b}$ : Under the assumption that the universe is dominated by dark matter at early times, the photon-baryon fluid oscillates under the driving force of gravity and the restoring force of photon pressure. The effective mass term of the photon-baryon fluid depends on the baryon-to-photon ratio. As the baryon contribution is enhanced, the additional mass provided by the baryons increases the compression of the fluid in the well and enlarges the compression peaks (odd peaks). In addition, the photons diffuse in the photon-baryon fluid hy an amount determined by the ionization history and the baryon content 31 . The effect of this diffusion is a damping of structure on small scales as photons from over dense regions mix with those from under

${ }^{a} \Omega_{D E}$ refers to a component of dark energy with negative presflep but in which the equation of state, $w>-1$ in contrast for that of $\Lambda$ for which $w=-1$ 目, 22 
dense regions. The extent to which the angular spectrum is suppressed at higher multipoles will, therefore depend on the baryon content.

- $H_{0}$ : During radiation domination, radiation pressure induces a decay of the gravitational potential, boosting the height of the acoustic peaks. If $H_{0}$ is relatively lower, matter-radiation equality happens later and the peaks are enhanced. In other words, one interpretation of a high first acoustic peak is a relatively lower value of the Hubble constant which implies a delay in matter-radiation equality. The location of the peaks also depends weakly on the Hubble constant. The value of the Hubble constant affects the distance to the last scattering surface. A larger distance to the last scattering surface will result in features of known physical extent appearing at smaller angular scales, which translates to a shift in the peaks to higher $l$.

The currently favored class of models are inflation-inspired adiabatic models in which potential wells influence all particle species in the same way; the fractional fluctuation in the number density of each species is independent of species. Most of the above discussion assumes models of this type. Isocurvature models, on the other hand, arise from initial conditions where there are no potential wells. Fluctuations in the photon density balance fluctuations in the other particle species in order to maintain zero curvature in the initial state. As in the adiabatic case, oscillations are produced in the photon-baryon fluid but they are $90^{\circ}$ out of phase with respect to those expected from adiabatic initial conditions. The first acoustic peak is therefore expected to be found at $l \approx 350 \Omega^{-1 / 2}$ rather than $l \approx 200 \Omega^{-1 / 2}$ as is expected for adiabatic models 31 . Isocurvature conditions can arise from cosmological defects (strings, monopoles, and textures) 41, 1ㅡ. An essential signature of most defect models is that temporal phase coherence for Fourier modes of a given wavenumber, which is responsible for driving the acoustic peaks, is destroyed by the non-linear evolution of the defect network. The result is that the fluctuation power is smeared out and the series of acoustic peaks is converted into a single broad peak, wider than the observed first Doppler peak and shifted to higher $l$. Simple isocurvature models are not consistent with contemporary CMB measurements.

\section{CMB Anisotropy Measurements - Experimental Challenges}

Many experiments have been designed to measure the anisotropy in the CMB. These measurements are difficult due to the small relative amplitude of the effect. Fluctuations exist at the level of a few parts in $10^{5}$ of $\mathrm{a} \approx 3 \mathrm{~K}$ signal that is already difficult to distinguish from atmospheric noise, emission from Galactic and extragalactic sources, and systematic errors inherent to the ex-

miller: submitted to World Scientific on November 27, 2018 
periments. A successful CMB experiment must have high sensitivity and low and well understood systematic errors.

There are three general approaches that have been taken in order to measure anisotropy in the CMB. The first is to make a map of the fluctuations and to extract the power spectrum from the map. Examples of mapmaking experiments include satellite experiments such as COBE 65, NASA's recently launched MAP, and ESA's upcoming Planck. Mapping has alsp been a popular technique for ballogn-borne experiments such as QMAP 15 , BOOMERanG 13, and MAXIMA 88. CMB maps are characterized by complex scan patterns, facilitating the comparison of each pixel (containing a temperature measurement and an associated uncertainty) with each neighboring pixel. Mapping instruments are generally made up of a single collecting telescope dish as a primary mirror with secondary and sometimes tertiary mirrors used to focus the radiation into the receiver. Receivers are single or multiple element radiometers generally constructed using HEMT 58 detectors at frequencies below $90 \mathrm{GHz}$ and bolometers at higher frequencies.

The second class of CMB experiments are those making use of a scanning or beam switching technique to compare signals at difference locations on the sky. These experiments are constructed in much the same way as are mapmaking instruments (also generally single-dish telescopes using HEMTs and bolometers) but the scan strategy is optimized for probing the power spectrum directly without the intermediate step of making a map. This is an approach often taken by ground-based experiments, simplifying the scan to a constant elevation in order to keep the atmospheric temperature as uniform as possible over the scan. Examples of this type of experiment are Saskatogn 6 , MSAM 20 , MAX 67 Tenerife 63, PYTHON 10 , VIPER 55, and MAT 44.71.

The third general class of CMB experiments are those using interferometry. Since the natural data products of an interferometer are components of the Fourier transform of the sky brightness distribution, interferometry is a natural way to measure the angular power spectrum of the CMB. Interferometers also have the advantage of low systematic errors, easy removal of point sources, and good atmospheric rejection. Interferometers require a phase-preserving detector so generally HEMT amplifiers or SIS mixers are used rather than the more sensitive bglometers. Examples of interferometric CMB experiments are CAT 3, DASI 40 , IAC29, and CBI 48 .

\section{Early CMB Experiments}

The Hot Big Bang model was introduced by Gamow in 194623 and the existence of a cosmic radiation background was first predicted by Alpher and

miller: submitted to World Scientific on November 27, 2018 
Herman in 1948 . These ideas were largely ignored, however until the sixties when Dicke and Peebles worked out a new hot big bang model and predicted the existence of a resulting thermal background relic. Roll and Wilkinson were preparing an experiment to search for this radiation when it was detected by Penzias and Wilson in 1964 as excess noise in their communications antenna. The discovery 53 and the explanation 16 were published in companion papers in 1965 and the field of CMB physics was officially on the map. The CMB spectrum was definitively measured over the frequency range between 70 and $630 \mathrm{GHz}$ by the Cosmic Background Explorer (COBE) Satellite's Far Infrared Absolute Spectrophotometer (FIRAS) instrument and found to be that of a thermal blackbody with a temperature of $2.728 \pm 0.004 \mathrm{~K}$ at the $95 \%$ confidence level 21 .

While the measurement of the CMB and the identification of the radiation as such a strikingly good black body was a triumph for the big bang model, information about the formation history of the large scale structure in the universe is contained not in the absolute measurement of the CMB but in measurements of the anisotropy. Had no anisotropy been detected, the entire model of structure formation growth by the collapse of density inhomogeneities would have to have been reformulated. The COBE DMR detection of these anisotropies on angular scales of several degrees 65 therefore marked another large milestone in the field and encouraged other experimentalists to look further.

COBE measured fluctuations on scales that reflect the primordial fluctuation field; those fluctuation modes that had not yet had time to complete an oscillation in the potential well before crossing the horizon. These measurements therefore normalize the CMB spectrum and can be used to connect with measurements of large scale structure.

Since the location in $l$-space of the first peak contains the most direct known measurement of the geometry of the universe and the location and height of this peak contain a wealth of information about other cosmological parameters, the next challenge was to determine the shape of the angular power spectrum on scales near the location of the predicted first peak. Since the COBE DMR detection, measurements of the CMB have largely focused on this goal. While descriptions of individual experiments will be confined to recently published results, it is important to note the immense amount of progress that was made by early experiments. Figure 1 shows CMB data published before 1999. It is clear that there is evidence for a rise in the spectrum up to $l \approx 200$ and a fall above $l \approx 400$ but no single instrument had detected and localized the peak. Since each instrument has a calibration error on the order of $10 \%$ or more and the error bars are large, measurements at different

miller: submitted to World Scientific on November 27, 2018 
angular scales made with different instruments are difficult to compare in a quantitative way. It was therefore important that single instruments (with the same systematic errors and the same calibration uncertainties) measured the angular spectrum over the entire range of multipoles spanning the predicted location of the peak.

\section{Modern CMB Experiments}

The dream of the CMB being used to place meaningful constraints on cosmological parameters has come true over the past couple of years. The location of the first Doppler peak has been conclusively measured, the second and arguably third peaks have been detected, and constraints on parameters have been inferred from the location and relative heights of these peaks. It is therefore useful to discuss in more detail these measurements and the instruments that have made them possible. Data from modern CMB experiments is plotted in Figures 2a-2c and Table 1 shows experimental parameters and the best fit cosmological models for each of the experiments.

The first detection of the first Doppler peak with a single instrument came from the Mobile Anisotropy Telescope on Cerro Toco (MAT/Toco) 4 , a single dish scanning experiment using HEMT 58 and SIS-based 35 receivers. MAT operated at the base of Cerro Toco mountain at an altitude of 17,000 ft. (5200 m) in the Atacama desert in Northern Chile. Data published in 1999 from this experiment (see Figure 2a) localized the peak in the spectrum to $l \approx 200$, giving support to adiabatic, inflationary models and ruling out the most common class of isocurvature and texture models. The amplitude of the first peak as measured by Toco was also suggestively high. As seen in figure 2 the data does not fit the standard CDM model very well and is a better fit to a $\Lambda$ CDM model.

The next experiment to detect a peak in the CMB power spectrum was the BOOMERanG experiment 13. BOOMERanG is also a single dish experiment with multiple detectors operating at higher frequency and higher angular resolution than the Toco experiment. It uses bolometers rather than HEMTs or SISs and operates from a balloon platform. BOOMERanG was the first CMB experiment to fly on a long duration balloon from Antarctica. Before flying in Antarctica, the instrument underwent a test flight in North America. Data from the test flight (BOOM/NA) 42 was published early in 2000 and showed striking agreement with the Toco data in the location of the peak (see Figures 2a and b). The amplitude of the peak is lower than that measured by Toco but agrees within the uncertainties. Also published in 2000, data from the BOOMERanG long duration flight (BOOM/LDB) 12

miller: submitted to World Scientific on November 27, 2018 
measured, for the first time, entire rang of $l$-space covering the predicted locations of the first and second peaks in the angular power spectrum. This data caused some speculation at the time of publication because while power was clearly detected in the region of the second peak, the amplitude was lower than predicted by the cosmological models that were at the time becoming standard.

Also published in 2000, data from the MAXIMA experiment again confirmed the location of the first peak and measured the region of $l$-space covering the second peak 27. MAXIMA 30 is a balloon-borne mapping experiment that flew from North America. While MAXIMA saw slightly more power in the region of the second peak than BOOMERanG did, joint fits to the MAXIMA and BOOMERanG data vielded a value of $\Omega_{b} h^{2}$ higher than predicted from Big Bang Nucleosynthesis 79 .6. Analyses suggested that within the standard cosmology and standard BBN, this value could not be accommodated and alternative theories were proposed such as non-standard reionization histories 28, and the possibility of evolution in the electromagnetic entropy since the time of BBN 34.

Data published in 2001 resolved this discrepancy. Three data sets were released in the same weekend, covering the range of $l$-space spanning the first three Doppler peaks, which agreed remarkably well. Figure 2c shows data from the first year of DASI observations along with re-anlyses of the BOOMERanG and MAXIMA 2000 data. Differences between the 2000 and 2001 analyses for these experiments are outlined in the caption for Figure 2.

DASI (Degree Angular Scale Interferometer) is a very different instrument from those discussed above. Rather than being a single dish mapping or scanning telescope, it is a compact interferometer designed specifically for the purpose of measuring anisotropy in the CMB. Like Toco, it uses HEMT amplifiers and operates from the ground (in this case the South Pole). DASI was designed to operate as a sister experiment to another interferometer, CBI (Cosmic Background Interferometer) with larger dishes and larger dish separation than DASI, therefore designed to probe smaller angular scales (see Figure 2c). CBI operates from the a desert plateau in the Northern Atacama desert very close to the Toco site.

Focusing on figure $2 \mathrm{a}-\mathrm{c}$, there are a couple of subtleties to note. First, the data plotted in figures 2a-2c do not include calibration uncertainties. These uncertainties are listed in parentheses in the upper right of each figure. One is free to shift each entire data set up or down by the calibration uncertainty. In addition, the BOOMERanG and MAXIMA data sets have uncertainties in the effective beam size that are not included in the plotted error bars. These uncertainties depend on $l$ (grow with increasing $l$ ) so the effect is to tilt the 
spectrum. See Netterfield et al. for an illustration of the amplitude of the tilt corresponding to the 1 sigma uncertainty in the BOOMERanG beam of $\pm 13 \%$. Beam and pointing errors for the MAXIMA experiment are listed as a function of $l$ in Lee $e t$ al. and range from $0 \%$ at $l=77$ to ${ }_{-18}^{+25} \%$ and $\pm 10.2 \%$ at $l=1147$ for beam and pointing respectively. Caveats stated, agreement between the three independent measurements covering a large range of angular scales and the CBI result with the smallest error bars at the smallest angular scale is impressive. In addition, it is reassuring to note that while BOOMERanG and MAXIMA are similar instruments employing similar scan strategies, DASI is a completely different type of instrument producing indistinguishable results.

Figure 2d shows CMB data as combined by Wang, Tegmark, and Zaldarriaga 4 . Data have been compressed from an original 105 bandpowers to the 24 bandpowers shown in the Figure 2d. Clearly evident are three peaks in the CMB power spectrum, the first of which appears at $l \approx 200$, followed by a decrease in power at smaller scales. Cosmological implications are discussed below.

\subsection{Cosmological Parameters and Implications}

As discussed above, much of the focus of CMB experiments following COBE has gone into characterizing the first Doppler peak in order to constrain the geometry of the universe. Using the Toco data and the BOOMERanG/NA data alone, Knox and Page performed a model-independent fit to the data solving for the location and height of the first Doppler peak 36, localizing it to be at $175<l<243$ (Toco) and $151<l<259$ (BOOM/NA) both at the $95 \%$ confidence level, as predicted by adiabatic inflationary models. Models predicting the peak location to be at $l \approx 350-400$ (isocurvature and texture models) are therefore ruled out at the many sigma level by each data set independently. Combining this data with recent low measurements of the matter density 22 we can infer that there must be a component of dark energy in the universe. This agrees well with supernova measurements 54,61, which suggest that the cosmological constant (or other form of dark energy) plays a significant role in the dynamics of the universe today.

Beyond the location of the first peak, the detection of the other peaks along with the characterization of their locations and heights relative to each other and to the first peak shed light on cosmological parameters. Table 1 shows the values of several cosmological parameters as measured by each of the CMB experiments both independently and when combined into a single set of bandpowers 4 . It is clear that individual determinations are consistent with each other and with the general picture of a flat, low $\Omega_{m}$ universe that

miller: submitted to World Scientific on November 27, 2018 
Table 1. Top: Experimental parameters References at top refer to papers describing the instruments. Bottom: Best fit cosmological parameters Column two shows the fit values for TOCO+DMR assuming a prior of $\Omega_{b} h^{2}=0.019 \pm 0.003$ and $h=0.65 \pm 0.118$. No formal errors were calculated. Column three shows cosmological parameters extracted from BOOM/LDB 2001 data. Error bars are one sigma and assumed priors are $0.45<h<0.9$, and age $>10 \mathrm{Gyr} 44$. Similar fits were done to the BOOM/LDB 2000 data assuming the same priors resulting in $\Omega_{T O T}=1.15_{-0.09}^{+0.1}, \Omega_{b} h^{2}=0.036_{-0.005}^{+0.006}, \Omega_{C D M} h^{2}=0.24_{-0.09}^{+0.08}$, $\Omega_{m}=0.84 \pm 0.29, \Omega_{\Lambda}<0.83$, and $n_{s}=1.04_{-0.09}^{+0.1} 37$. Fits to BOOM/NA data result in $0.85<\Omega_{T O T}<1.25$ at the $68 \%$ confidence level and when combined with CPRE results in a best fit model of $\Omega_{C D M}=0.46, \Omega_{b}=0.05, \Omega_{\Lambda}=0.5, n_{s}=1$, and $h=7043$. Column four shrpws the best fit parameters for the MAXIMA data released in 2000 combined with COBE 2. Error bars are at the $95 \%$ confidence level with $0.4<h<0.9$, age $>10 \mathrm{Gyr}$, $\Omega_{m}>0.1$. The values of $\Omega_{\Lambda}$ and $\Omega_{m}$ were calculated combining MAXIMA data with Supernova results. Under the same assumptions, fits to the MAXIMA 2001 data combined with COBE result in (at the $95 \%$ confidence level) $\Omega_{b} h^{2}=0.0325 \pm 0.0125, \Omega_{C D M} h^{2}$ $0.17_{-0.07}^{+0.16}, \Omega_{T O T}=0.9_{-0.16}^{0.18}$, and $n_{s}=0.46 \tau_{c}=(0.99 \pm 0.14)$ assuming that $\tau_{c} \leq 0.566$. Column five contains the best fit cosmological parameters to the DASI data 59 . Errors are $68 \%$ confidence and priors are $h>0.45$ and $0.0<\tau_{c} \leq 0.4$. Constraints on $\Omega_{m}$, and $\Omega_{\Lambda}$ also assume $h=0.72 \pm 0.08$. Column six tabulates the best fit parameters from combined bandpowerd 74 . Fits are to the CMB data alone with $95 \%$ confidence limits.

\begin{tabular}{|l|ccccc|}
\hline & Tocd 45 & BOOM 3 & MAXIMA3 & DASI电 & Comb. \\
\hline Platform & ground & balloon & balloon & ground & - \\
Type & beam-switch & mapping & mapping & int & - \\
Detector & HEMT,SIS & Bolo & Bolo & HEMT & - \\
Freq (GHz) & $30-144$ & $90-400$ & $150-410$ & $26-36$ & - \\
resolution & $0.2^{\circ}-1^{\circ}$ & $0.17^{\circ}-0.23^{\circ}$ & $10 \prime$ & $20 \prime$ & - \\
Sky (deg) & 500 & 1800 & 124 & 400 & - \\
\hline$\Omega_{T O T}$ & - & $1.03 \pm 0.06$ & $0.90 \pm 0.15$ & $1.04 \pm 0.06$ & - \\
$\Omega_{K}$ & 0 & - & - & - & $-0.06_{-0.59}^{+.13}$ \\
$\Omega_{m}$ & - & $0.50 \pm 0.20$ & $0.25-0.5$ & $0.4 \pm 0.15$ & - \\
$\Omega_{\Lambda}$ & 0.57 & $0.52_{-0.19}^{+0.24}$ & $0.4-0.76$ & $0.6 \pm 0.15$ & $0.60_{-0.55}^{+0.32}$ \\
$\Omega_{C D M} h^{2}$ & 0.16 & $0.12 \pm 0.05$ & $0.13 \pm 0.10$ & $0.14 \pm 0.04$ & $0.10^{+0.07}$ \\
$\Omega_{\mathrm{b}} h^{2}$ & 0.019 & $0.021_{-0.003}^{+0.004}$ & $0.025 \pm 0.01$ & $0.022_{-0.003}^{+0.004}$ & $0.02_{-0.01}^{+0.06}$ \\
$n_{S}$ & 1.18 & $0.93_{-0.08}^{+0.1}$ & $0.99 \pm 0.09$ & $1.01_{-0.06}^{+0.08}$ & $0.93_{-0.16}^{+0.75}$ \\
\hline
\end{tabular}

is currently dominated by some form of dark energy.

\section{The Future of the CMB}

While tremendous progress has been made using the CMB to measure cosmological parameters, much remains to be done. In terms of primary anisotropies the error bars and calibration uncertainties over the range of measured angular scales need to be reduced, the detailed shape of the damping tail at 
small angular scales has yet to be characterized, and the range of angular scales between the COBE measurement and the more recent measurements have yet to be connected by a single instrument. In addition, more work needs to be done in order to fully understand the extent to which foregrounds such as point sources, dust, and synchrotron radiation are capable of contaminating CMB results. Other foregrounds such as the Sunyaev-Zel'dovich effect (SZE) are capable of providing powerful probes of the early universe in their own right. Furthermore, the same processes responsible for creating the temperature anisotropies discussed throughout this paper should also produce polarization anisotropies. The spectrum of these anisotropies will provide a complementary look at the early universe and will allow the removal of degeneracies inherent to measurement of the temperature anisotropy spectrum alone.

Experiments designed to measure primary anisotropies, polarization anisotropies, and the Sunyaev-Zel'dovich effect are underway. NASA's MAP satellite, launched on June 30th, 2001 will provide detailed information about the spectrum of primary anisotropies out to $l \approx 1000$. It is also expected to detect the polarization of the CMB over a wide range of multipoles. ESA's Planck satellite, scheduled for launch in 2007 will measure the spectrum of both primary and polarization anisotropies up to $l \approx 2000$. Planck also expects to survey the Sunyaev-Zel'dovich effect in clusters of galaxies. In the mean time, several groups are analyzing data already obtained and many balloon-based and ground-based experiments are being designed to measure CMB polarization and to conduct non-targeted SZE surveys. Detector technology continues to improve and new techniques are rapidly being developed. The near future in this field promises to be as exciting as the recent past.

\section{Acknowledgments}

The author would like to thank Erik Leitch for a thorough reading of the text, Mark Devlin and Andreas Berlind for helpful comments, and is supported by Hubble Grant ASTR/HST-HF-0113.

\section{References}

1. Alpher, R. A., and Herman, R. C., Nature, 162, 774 (1948).

2. Balbi, A., et al., ApJ, 545, L1-L4; Erratum-ibid. 558, L145-L146 (2000).

3. Baker, J. C., et al., MNRAS, 308, 1173 (1999).

4. Barreiro, R. B., New Astron.Rev. 44 179-204 (2000).

5. Bond, J. R., Phys. Rev. Lett., 74, 4369 (1995). 
6. Bond, J. R., et al., Proc. IAU Symposium, 201 (PASP), CITA-2000-65 (2000).

7. Burles, S., Nollett, K., and Turner, M., Phys. Rev. D, 63, 063512 (2001).

8. Caldwell, R. R., Dave, R., Steinhardt, P. J., 1998, Phys. Rev Lett., 80, $1582(1998)$.

9. Coble, K, PhD Thesis, The University of Chicago (1999).

10. Coble, K., et al., ApJ, 519, L5 (1999).

11. Crittenden, R. G., \& Turok, N. G., Phys. Rev Lett., 75, 2642 (1995).

12. de Bernardis, P., et al., Nature, 404, 955-959 (2000).

13. de Bernardis, P., et al., astro-ph/9911461 (1999).

14. de Oliveira-Costa, A., Devlin, M. J., Herbig, T., Miller, A. D., Netterfield, C. B., Page, L. A., and Tegmark, M., ApJ, 509, L77 (1998).

15. Devlin, M. J., de Oliveira-Costa, A., Herbig, T., Miller, A. D., Netterfield, C. B., Page, L., \& Tegmark, M., ApJ, 509, L73 (1998).

16. Dicke, R. H.; Peebles, P. J. E.; Roll, P. G.; Wilkinson, D. T., ApJ 142, 414-419 (1965).

17. Dicker, S., et al., MRAS, 309, 750 (1999).

18. Dodelson, S., private communication, (2000).

19. Dodelson, S., and Knox, L., Phys. Rev. Lett., 84, 3523 (2000).

20. Fixsen, D. J., et al., ApJ, 470, 63 (1996).

21. Fixsen, D. J., et al., ApJ, 473, 576 (1996).

22. Freedman, W. L., Phys. Scripta T85, 37-46 (2000).

23. Gamow, G., Phys Rev., 71, 273 (1946).

24. Gundersen, J., et al., ApJ, 443, L57-L60 (1995).

25. Gutiérrez, C. M., Rebolo, R., Watson, R. A., Davies, R. D., Jones, A. W., and Lasenby, A. N., ApJ, 529, 47 (2000).

26. Halverson, N., et al, astro-ph/0104489 (2001).

27. S. Hanany, et al., ApJ, 545, L5 (2000).

28. Hannestad, S., and Scherrer, R., astro-ph/0011188 (2000).

29. Harrison, D. L., et al., astro-ph/0004357 (2000).

30. Hu, W., PhD Thesis, The University of California at Berkeley (1995).

31. Hu, W., Sugiyama, N., \& Silk, J., Nature, 386, 37 (1997).

32. Huey, G., Wang, L., Dave, R., Caldwell, R. R., \& Steinhardt, P. J., Phys. Rev D, 59 (1998).

33. Jungman, G., Kamionkowski, M., Kosowsky, A., and Spergel, D., Phys.Rev.Lett., 76, 1007-1010 (1996).

34. Kaplinghat, M., and Turner, M., Phys. Rev. Lett., 86, 385 (2001).

35. Kerr, A. R., Pan, S. K., Lichtenberger, A. W., LLoyd, F. L, \& Horner, N., in 4th International Symposium on Space Terahertz Technology (1993).

36. Knox, L., \& Page, L., Phys. Rev. Lett., 85, 1366-1369 (2000). 
37. Lange, A., et al., Phys. Rev. D, 63, 042001 (2001).

38. Lee, A., et al., in EC-TMR Conference Proceedings 476, 3K Cosmolgy, ed. L. Maiani, F. Melchiorro, and N. Vittorio (Woodburym New York: AIP, 224, 1999).

39. Lee, A., et al, ApJ, 561, L1-L5 (2001).

40. Leitch, E. M., Readhead, A. C. S., Pearson, T. J., Myers, S. T., Gulkis, S., and Lawrence, C. R., ApJ, 532, 37 (2000).

41. Magueijo, J., \& Brandenberger, R., astro-ph/0002030 (2000).

42. Mauskopf, P., et al., ApJ, 536, L59-L62 (2000).

43. Melchiorri, A., et al., ApJ, 536, L63-L66 (2000).

44. Miller, A. D., et al., ApJ, 524, L1-4 (1999).

45. Miller, A. D., in preparation, astro-ph/0108030 (2001)

46. Netterfield, C. B., Devlin, M. J., Jarosik, N., Page, L., and Wollack, E. J., ApJ, 474, 47 (1997).

47. Netterfield, C. B., et al., astro-ph/0104460 (2001).

48. Padin, S., et al., astro-ph/0110124(2001).

49. Padin, S., et al., ApJ 549 L1-L5 (2001).

50. Page, L. A., Conference Proceedings, astro-ph/9911199 (1999).

51. Partridge, B., 3K: The Cosmic Microwave Background, (Cambridge University Press, Cambridge, 1995).

52. Peacock, J. A., Cosmological Physics, (Cambridge University Press, Cambridge, 1999).

53. Penzias, A. A., and Wilson, R. W., ApJ, 142, 419-421 (1965).

54. Perlmutter, S., et al., Bull Am. Astron. Soc., 29, 1351 (1997).

55. Peterson, J. B., et al., astro-ph/9910503 (1999).

56. Piccirillo, L., and Calisse, P., ApJ, 411, 529 (1993).

57. Podariu, S., Souradeep, T., Gott, R., Ratra, B., amd Vogeley, M., Ap.J. 559, 9 (2001).

58. Pospieszalski, M. W., Weinreb, S., Norrod, R. D., \& Harris, R., IEEE Trans. Microwave Theory Tech., MTT-36, 3, 552 (1998).

59. Pryke, C., et al., asro-ph/0104490 (2001).

60. Ratra, B., Ganga, K., Stompor, R., Sugiyama, N., de Bernardis, P, and Górski, K. M., ApJ, 510, 11 (1999a).

61. Riess, A., et al., Astron. J., 116, 1009-1038 (1998).

62. Rocha, G., Stompor, R., Ganga, K., Ratra, B., Platt, S. R., Sugiyama, N., and Górski, K. M., ApJ, 517, 549 (1999b).

63. Romeo, G., Ali, S., Femenia, B., Limon, M., Piccirillo, L., Rebolo, R., and Schaefer, R., ApJ, 548, L1-L4 (2000).

64. Scott, P. F., et al., ApJ, 461, L1 (1996).

65. Smoot, G., et al., ApJ, 396, L1 (1992).

miller: submitted to World Scientific on November 27, 2018 
66. Stompor, R., et al., astro-ph/0105062 (2001).

67. Tanaka, S. T., et al., ApJ, 468, L81 (1996).

68. Tegmark, M, "Doppler Peaks and all that: CMB Anisotropies and what they can tell us". Proc. Enrico Fermi, Course CXXXII, (Varenna, 1995).

69. Tegmark, M., \& Hamilton, astro-ph/9702019 (1997).

70. Tegmark, M., and Zaldarriaga, M., Phys. Rev. Lett., 85, 2240 (2000).

71. Torbet, E. T., et al., ApJ, 521, L79 (1999).

72. Tucker, G. S., Gush, H. P., Halpern, M., and Shinkoda, I., ApJ, 475, L73 (1997).

73. Seljak, U., and Zaldarriaga, M., Ap. J., 469, 437 (1996).

74. Wang, X., Tegmark, M., and Zaldarriaga, M., astro-ph/0105091 (2001).

75. Wilson, G. W., et al., ApJ, 532, 57 (2000).

76. Wollack, E. J., Jarosik, N., Netterfield, C. B., Page, L., \& Wilkinson, D., Ap. J., 419, L49 (1993). 

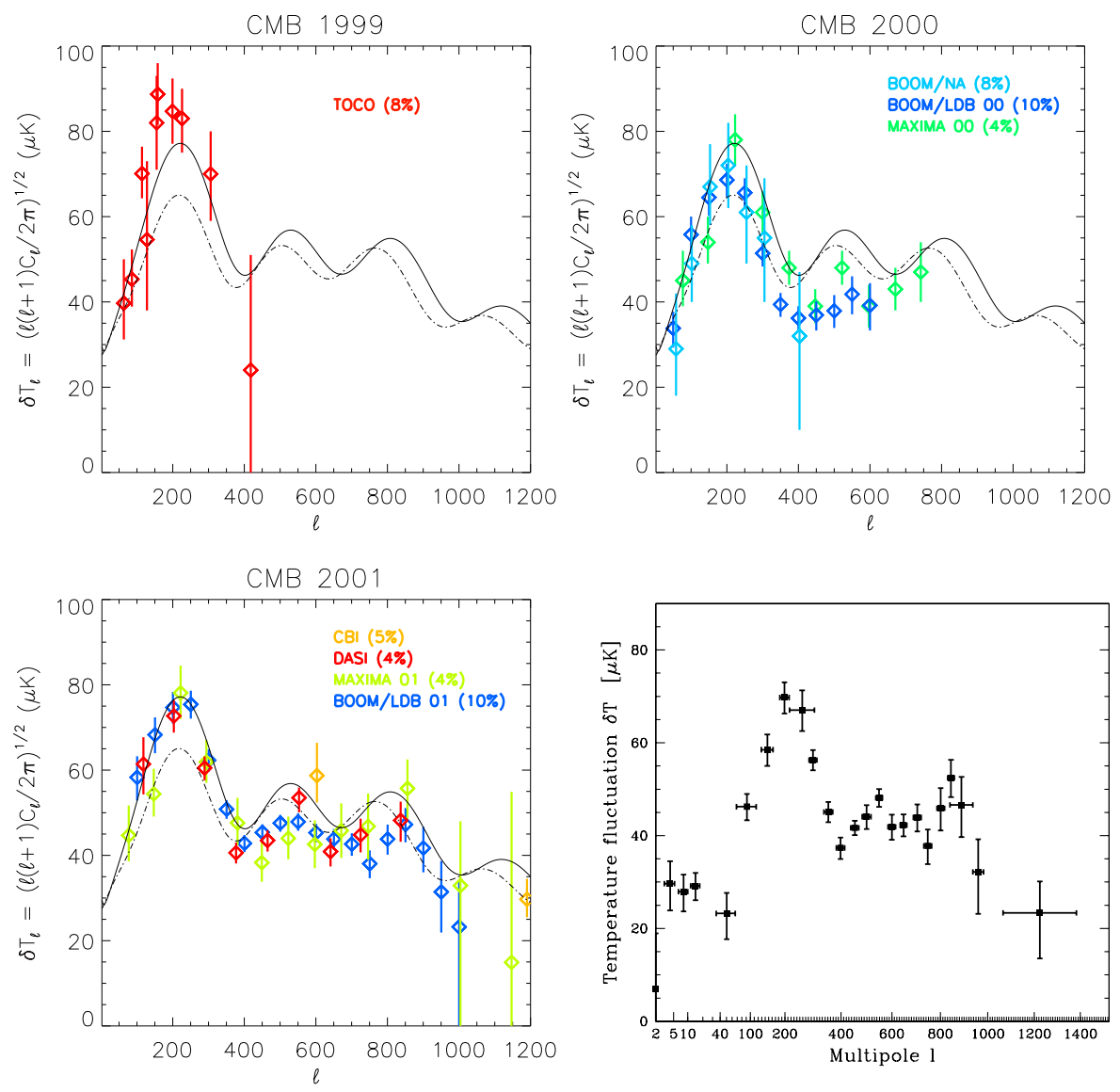

Figure 2. a (upper left): The MAT/TOG data 44 . 71 b (upper right):Data frem BOPA/NA 42 , BOOM/LDB 12 , and MAXF1A27 published in 2000. c (lower left) PASIE6, CBI49, the BOOM/LDB 2001 analysi 47, and the MAXIMA 2001 analysis 39 . The BOOM/LDB 2001 analysis differs from the 2000 analysis in that the number of channels analyzed were increased from one to four, the data from the second half of the flight was added, the effective beam size was atjusted from $10.0 \pm 0.1$ ' to $12.9 \pm 1.4$, and the overall calibration was raised by $10 \%$ in $C_{l} 4$. The MAXIMA 2001 analysis differs from the 2000 analysis in that, the number of channels used was dropped from four to three (the $240 \mathrm{GHz}$ channel was removed leaving three channels at $150 \mathrm{GHz}$ ). Also, the 2000 analysis made use of the entire map binned into pixels of size $5 / \times 5 /$ while in 2001 analysis was restrict to the central portion of the map and the data was re-binned into pixels of size $3 / \times 3 / 66$. d (lower right) $\mathrm{CMB}$ combined bandpowers 74 obtained by combining much of the data from Figure 1 and all data from Figure 2 with the exception of BOOM/LDB 2000 and MAXIMA 2000. Combined bandpowers include calibration and beam uncertainties from individual experiments. Note, models in each panel are the two $\Omega_{T O T}=1$ models from Figure 1 and numbers in parentheses are calibration uncertainties.

miller: submitted to World Scientific on November 27, 2018 